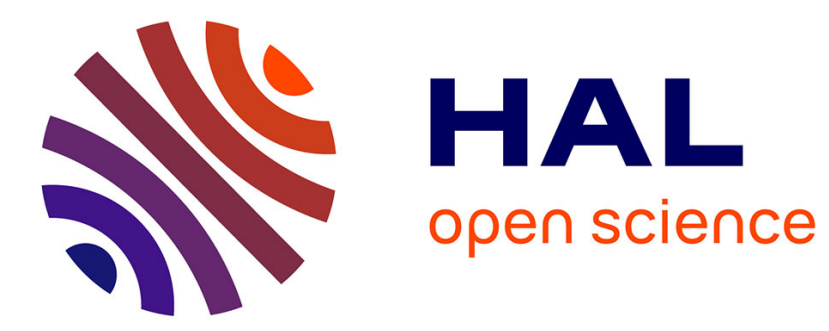

\title{
Hurst exponents and delampertized fractional Brownian motions
}

\author{
Matthieu Garcin
}

\section{To cite this version:}

Matthieu Garcin. Hurst exponents and delampertized fractional Brownian motions. 2018. hal01919754

\section{HAL Id: hal-01919754 \\ https://hal.science/hal-01919754}

Preprint submitted on 12 Nov 2018

HAL is a multi-disciplinary open access archive for the deposit and dissemination of scientific research documents, whether they are published or not. The documents may come from teaching and research institutions in France or abroad, or from public or private research centers.
L'archive ouverte pluridisciplinaire HAL, est destinée au dépôt et à la diffusion de documents scientifiques de niveau recherche, publiés ou non, émanant des établissements d'enseignement et de recherche français ou étrangers, des laboratoires publics ou privés. 


\title{
Hurst exponents and delampertized fractional Brownian motions
}

\author{
Matthieu Garcin*
}

November 12, 2018

\begin{abstract}
The inverse Lamperti transform of a fractional Brownian motion is a stationary process. We determine the empirical Hurst exponent of such a composite process with the help of a regression of the log absolute moments of its increments, at various scales, on the corresponding log scales. This perceived Hurst exponent underestimates the Hurst exponent of the underlying fractional Brownian motion. We thus encounter some time series having a perceived Hurst exponent lower than $1 / 2$, but an underlying Hurst exponent higher than $1 / 2$. This paves the way for short- and medium-term forecasting. Indeed, in such series, mean reversion predominates at high scales, whereas persistence is overriding at lower scales. We propose a way to characterize the Hurst horizon, namely a limit scale between these opposite behaviours. We show that the delampertized fractional Brownian motion, which mixes persistence and mean reversion, is relevant for financial time series, in particular for high-frequency foreign exchange rates. In our sample, the empirical Hurst horizon is always above 1 hour and 23 minutes.
\end{abstract}

Keywords - fractional Brownian motion, Hurst exponent, Lamperti transform, Ornstein-Uhlenbeck process, foreign exchange rates

\section{Introduction}

The fractional Brownian motion (fBm) was introduced by Mandelbrot and van Ness [25]. With a Hurst exponent $H \in(0,1)$ and a volatility parameter $\sigma$, the $\mathrm{fBm} X$ is defined as the only zeromean Gaussian process with zero at the origin and with the following covariance function, for all $(s, t) \in \mathbb{R}^{2}:$

$$
\mathbb{E}\left\{X_{t} X_{s}\right\}=\frac{\sigma^{2}}{2}\left(|t|^{2 H}+|s|^{2 H}-|t-s|^{2 H}\right) .
$$

By definition, $X$ is $H$-self-similar: $\forall t, \lambda>0, X_{t}$ and $\lambda^{-H} X_{\lambda t}$ have the same probability distribution. An important fact is that any $H$-self-similar process is derived from a stationary process $S$, thanks to the Lamperti transform of $S$, noted $\mathcal{L}_{H} S$. This transformation is invertible. Therefore, the inverse Lamperti transform of the $\mathrm{fBm} X$ or of any $H$-self-similar process, noted $\mathcal{L}_{H}^{-1} X$, is a stationary process [21, 14. For $t \in \mathbb{R}$, the Lamperti transform of $S$ is defined by $\left(\mathcal{L}_{H} S\right)_{t}=t^{H} S_{\ln (t)}$ and the inverse Lamperti transform of $X$ by $\left(\mathcal{L}_{H}^{-1} X\right)_{t}=e^{-H t} X_{\exp (t)}$. The Lamperti transform is convenient, as it allows using well-established tools from the framework of stationary processes for

*Léonard de Vinci Pôle Universitaire, Research center, 92916 Paris La Défense, France, matthieu.garcin@m4x.org. 
problems related to self-similar processes. For instance, tests of stationarity can be used to define self-similarity tests 22 .

The delampertized $\mathrm{fBm}, \mathcal{L}_{H}^{-1} X$, is thus a stationary process built from a fBm. However, delampertizing is not the only way to stationarize a $\mathrm{fBm}$. Indeed, the Ornstein-Uhlenbeck process, which is a standard example of stationary process, can be generalized to the fractional Ornstein-Uhlenbeck (fOU) process [19]. The fOU process is stationary, thanks to a mean reversion effect that appears explicitly in the equation defining this process.

It is worth noting that the delampertized fBm does not share with the fBm exactly the same properties. Neither does it with the fOU process, even though both are stationary. Among all these properties, two seem particularly relevant to understand and interpret the parameters of the delampertized fBm:

$\triangleright$ How is statistically driven the stationarity of the delampertized fBm?

$\triangleright$ What does the estimated Hurst exponent of a delampertized fBm tell about the properties of this process?

These questions are pivotal when facing the statistical study of fractional dynamics. Indeed, in the empirical literature, the analysis of fractional dynamics is mostly restricted to the framework of the $\mathrm{fBm}$. The guideline is usually the following: assuming that the $\mathrm{fBm}$ is well specified for the observed series, the Hurst exponent is estimated and decisions are taken accordingly. Indeed, for a $\mathrm{fBm}$, a Hurst exponent above (respectively below) 1/2 means persistence (resp. anti-persistence) of the increments. Therefore, in fields as diverse as finance [11, 27, 15, 20] or medicine [33, 34, researchers hunt for values of Hurst exponents above $1 / 2$, in order to leverage the persistence of the series and make predictions. Or, on the contrary, values of Hurst exponents below $1 / 2$ are expected to make us benefit from the mean reversion of the series, as in pair trading [32]. However, the good specification of the $\mathrm{fBm}$ model in these practical examples is rarely challenged. We believe that, for time series of interest rates, volatilities, or foreign exchange rates, a stationary delampertized $\mathrm{fBm}$ would be more appropriate than a simple geometric $\mathrm{fBm}$, which is very unlikely to remain between bounds. Then, the estimator for the Hurst exponent of the underlying fBm should be different in these two models and, for a same estimated value, different conclusions regarding the persistence of the series could arise between the fBm case and the delampertized fBm case.

The relevance of these questions is confirmed by the findings presented in this paper. In particular, we show that estimating the Hurst exponent of a delampertized fBm with the standard absolute-moment method designed for the $\mathrm{fBm}$ leads to a bias. A fBm-based method would indeed underestimate the Hurst exponent of a delampertized $\mathrm{fBm} \|^{1}$ The interpretation of the estimated Hurst exponent is tricky as well, since a value above $1 / 2$ does not necessarily mean persistence.

We define several notions of Hurst exponents. First, the perceived Hurst exponent is simply the Hurst exponent estimated as if the series was a $\mathrm{fBm}$, using a standard regression across scales. The underlying Hurst exponent is the Hurst exponent of the $\mathrm{fBm}$ before its delampertization. For a dynamics with an underlying Hurst exponent above $1 / 2$, the persistent aspect predominates at small scales, whereas the mean reversion is overriding at higher scales. The Hurst horizon is an approximation of the scale separating these two opposite sides.

We apply the delampertized fBm to financial data. We indeed found one other paper proving empirically, with the Hurst approach, that some financial time series are persistent at low scales and anti-persistent at higher scales [23. We confirm here this assertion for high-frequency foreign exchange rates and we provide a mathematical framework for this stylized fact. We explain why

\footnotetext{
1 That is to say, it will underestimate the Hurst exponent of the underlying fBm.
} 
the delampertized $\mathrm{fBm}$ is relevant for our sample, in particular more than the fOU process. We also show that the perceived Hurst exponent is almost always below $1 / 2$, whereas the underlying Hurst exponent is above this threshold. This makes predictions feasible up to the Hurst horizon, which has a magnitude of several hours, whereas such series were reputed to be unpredictable due to their perceived Hurst exponent. We also study in more detail the case of GBP/EUR, which has a very long Hurst horizon of several days. We analyse as well the impact of the Brexit referendum on our estimations.

The rest of the paper is organized as follows. Section 2 addresses the question of the mean reversion mechanism in the delampertized fBm. Section 3 is about the interpretation of the Hurst exponent of the underlying $\mathrm{fBm}$ in its delampertized version. Section 4 applies this mathematical framework to financial time series. Section 5 concludes.

\section{Mean reversion}

The $\mathrm{fBm}$ is a stochastic process with stationary increments, but the process itself is not stationary. The standard Brownian motion, as a particular case of the $\mathrm{fBm}$, has the same property. The Ornstein-Uhlenbeck process is a convenient way to define a stationary process using the nonstationary standard Brownian motion. It is based on an explicit mean reversion, characterized by a parameter depicting how intense the mechanism is. Substituting the standard Brownian motion by the fBm transforms the Ornstein-Uhlenbeck process in a fOU process, which is stationary as well. A fOU process $Z$ is defined by the following equation:

$$
d Z_{t}=-\alpha\left(Z_{t}-\mu\right) d t+\sigma d X_{t}
$$

where $X$ is a $\mathrm{fBm}, \sigma>0$ is the volatility parameter, $\mu \in \mathbb{R}$ is the long-term average parameter, and $\alpha>0$ is the speed of reversion. The parameter $\alpha$ and the corresponding mean reversion effect explain how a non-stationary process can be transformed in a stationary one. Indeed, when $Z_{t}$ is above (resp. below) the long-term average $\mu$, a negative (resp. positive) drift $-\alpha\left(Z_{t}-\mu\right)$ tends to attract $Z$ towards $\mu$. Such effect is balanced by $\sigma d X_{t}$, so that the intensity of the mean reversion is well illustrated by $\alpha / \sigma$. Of course, the farther $Z_{t}$ is from $\mu$, the more intense is the mean reversion. The fOU has been used for instance for modelling temperature [4, fluid turbulence [8] or the volatility of financial asset prices $[9]$.

Nevertheless, the fOU process is not the only way to model stationarity with the help of the fBm. Another solution is based on the inverse Lamperti transform. It is obtained by an exponential contraction of the time and an appropriate scaling in the state space. The inverse Lamperti transform of the $\mathrm{fBm} X$, of Hurst exponent $H$, is indeed defined by $\left(\mathcal{L}_{H}^{-1} X\right)_{t}=e^{-H t} X_{\exp (t)}$. Moreover, for $\theta>0$, the process $Y$, defined by

$$
Y_{t}=\left(\mathcal{L}_{H}^{-1} X\right)_{\theta t}
$$

is stationary as well.

A natural question arises: is the delampertized $\mathrm{fBm}$ the same as a fOU process? Both are indeed stationary and based on a fBm. In addition, if the Hurst exponent of the $\mathrm{fBm} X$ is $H=1 / 2$, which corresponds to the case of a standard Brownian motion, $\mathcal{L}_{H}^{-1} X$ and the standard OrnsteinUhlenbeck process based on $X$ are the same [6]. Nevertheless, the likeness stops here. Indeed, if $H \neq 1 / 2$, the autocovariance function of $\mathcal{L}_{H}^{-1} X$ decays exponentially, whereas the autocovariance function of the corresponding fOU process is a power function [6]. As a result, when $H \neq 1 / 2$, the delampertized $\mathrm{fBm}$ is not a fOU process. 
The equation defining the delampertized $\mathrm{fBm}$ is not based on an explicit mean reversion, contrary to the fOU process. Nevertheless, as a stationary process, a mean reversion exists in some way, even if it is only implicitly stated. We now want to depict more explicitly this mechanism. In the following theorem, we show that the delampertized $\mathrm{fBm}$ is indeed based on a mean reversion like the fOU process, despite the differences between the two processes. If $Y$ is the delampertized fBm, we show that any increment of $Y$ is negatively correlated to $Y_{s}$ itself, for $s$ before the observed increment. This assertion is true whatever $H$ and depicts the reversion effect toward 0 implied by this process. On the contrary, the sign of this covariance depends on $H$ in the case of a fBm.

Theorem 1. Let $H \in(0,1), 0<s \leq t<u$ and $X$ be a fBm of Hurst exponent $H$.

1. For the fBm $X$, we have:

$$
\operatorname{Cov}\left(X_{s}, X_{u}-X_{t}\right)=\frac{\sigma^{2}}{2}\left(u^{2 H}-t^{2 H}-(u-s)^{2 H}+(t-s)^{2 H}\right) .
$$

This covariance is strictly positive for $H>1 / 2$, strictly negative for $H<1 / 2$, and equal to zero for $H=1 / 2$.

2. Let $\theta>0$ and $Y_{t}=\left(\mathcal{L}_{H}^{-1} X\right)_{\theta t}$. Then:

$$
\operatorname{Cov}\left(Y_{s}, Y_{u}-Y_{t}\right)=\frac{\sigma^{2}}{2}[h(\theta(u-s))-h(\theta(t-s))],
$$

where $h: x \geq 0 \mapsto 2 \cosh (H x)-\left(2 \sinh \left(\frac{x}{2}\right)\right)^{2 H}$. This covariance is strictly negative, whatever $H \in(0,1)$.

The proof of this theorem, as well as of other results, is reported in appendix.

Mean reversion is verified statistically, for a process of mean 0 , when an increment is negatively correlated to a previous state of the process. A mean-reverting process is not necessarily stationary but, by essence, a non-constant stationary process should be mean-reverting. For instance, in Theorem 1. we see that a $\mathrm{fBm}$ of Hurst exponent strictly lower than $1 / 2$ is statistically meanreverting, whereas it is not stationary. Also, as we can expect from a stationary process, the delampertized $\mathrm{fBm} Y$ is statistically mean-reverting, whatever the value of $H$.

In addition, Theorem 1 provides us with more details about how this mean reversion works. We see, indeed, that the covariance of an increment with a previous state of $Y$, distant of a time horizon $\tau$, decreases from 0 to $-\sigma^{2} h(\theta \tau) / 2$ for a duration of the increment varying from 0 to infinity. For an increment of a given duration $\delta$, this covariance is asymptotically zero when the reference time $s$ is far in the past, but it decreases until $-\sigma^{2}(2-h(\theta \delta)) / 2$ when the reference time becomes closer to the beginning of the increment.

Until now, there is no surprise: the stationary process obtained by delampertizing a $\mathrm{fBm}$ is statistically mean-reverting and we gave some details about this effect. This result holds whatever the value of $H$. We can mention that the slope of the function $h$, in Theorem 1 , is steeper when $H$ is lower, what means that the covariance $\operatorname{Cov}\left(Y_{s}, Y_{u}-Y_{t}\right)$ is greater, in absolute value, for a lower $H$. In other words, the mean reversion is stronger for a lower $H$. Contrary to the $\mathrm{fBm}$, two distinct values of $H$ do not represent distinct features of the process, but they only depict a varying intensity in the same feature, at least regarding Theorem 1. Nevertheless, as we will see in the next section, when considering the correlation between increments, instead of between an increment and a reference state of the process, opposite interpretations about the persistence of the process can appear depending on the value of $H$. 


\section{About Hurst exponents}

The main purpose of this paper is to stress the fact that the Hurst exponent estimated directly on a time series $Y$ does not necessarily reflect the Hurst exponent of the underlying fBm $X$, if the time series is obtained by a transformation of $X$, in particular by an inverse Lamperti transform of $X$. In order to avoid any confusion, we define more precisely the two kinds of Hurst exponents we will work with:

$\triangleright H$ is the underlying Hurst exponent of $Y$ : it is the Hurst exponent of the fBm $X$ from which $Y$ is the inverse Lamperti transform.

$\triangleright$ If $\hat{H}(X)$ is an estimator of the Hurst exponent of the $\mathrm{fBm} X$, the same estimator applied to $Y$ instead of $X$, that is to say $\hat{H}(Y)$, provides us with an estimate of the perceived Hurst exponent of $Y$. The perception depends on the scale, or on the range of scales, used in the estimation.

The meaning of the Hurst exponent also varies between $X$ and $Y$. For example, a fBm with a Hurst exponent above $1 / 2$ is persistent, that is to say increments are positively autocorrelated. But the inverse Lamperti transform $Y$ of the same fBm does not have systematically positively autocorrelated increments. This doesn't mean that the perceived Hurst exponent, estimated directly on the series $Y$, is more appropriate to interpret the persistence of the series. In fact, estimating the Hurst exponent directly on $Y$ is meaningless, since the fBm model is not well specified for $Y$.

In this section, we will first discuss challenges linked to the estimation of Hurst exponents, then we will see how the underlying Hurst exponent can be interpreted.

\subsection{Estimation of underlying and perceived Hurst exponents}

The process $X$ is still a fBm, of Hurst exponent $H$, and $Y$, defined by equation (2), is a delampertization of $X$.

In this subsection, we adopt a statistical point of view. Therefore, the process $Y$ is now assumed to be observed in discrete time in the interval $[0, T / N]$, with a time step $1 / N$, where $N \in \mathbb{N}$. For $k \in \mathbb{N}$, let $M_{k, N, T}(X)$ and $M_{k, N, T}(Y)$ be the empirical absolute moment of order $k$ of the increments of $X$ :

$$
M_{k, N, T}(X)=\frac{1}{T} \sum_{i=1}^{T}\left|X_{i / N}-X_{(i-1) / N}\right|^{k}
$$

and of the increments of $Y$ :

$$
\begin{aligned}
M_{k, N, T}(Y) & =\frac{1}{T} \sum_{i=1}^{T}\left|Y_{i / N}-Y_{(i-1) / N}\right|^{k} \\
& =\frac{1}{T} \sum_{i=1}^{T}\left|e^{-\theta H i / N} X_{\exp (\theta i / N)}-e^{-\theta H(i-1) / N} X_{\exp (\theta(i-1) / N)}\right|^{k}
\end{aligned}
$$

For the fBm, among a rich litterature about estimators of Hurst exponents [26, 1, 24, 31, we know a simple estimator of $H$ based on absolute moments [29, 10, 15]:

$$
\check{H}_{k, N, T}(X)=-\frac{\log \left(\sqrt{\pi} M_{k, N, T}(X) /\left[2^{k / 2} \Gamma\left(\frac{k+1}{2}\right) \sigma^{k}\right]\right)}{k \log (N)},
$$

which converges almost surely towards $H$, when $T \rightarrow+\infty$. However, this estimator depends on the explicit knowledge of $\sigma$. If the volatility parameter $\sigma$ is unknown, one can define another estimator 
that is consistent with the moment-based estimators at two different scales, say for time steps $1 / N$ and $2 / N$ :

$$
\hat{H}_{k, N, T}(X)=\frac{1}{k} \log _{2}\left(\frac{M_{k, \frac{N}{2}, \frac{T}{2}}(X)}{M_{k, N, T}(X)}\right) .
$$

Like $\check{H}_{k, N, T}(X)$, the estimator $\hat{H}_{k, N, T}(X)$ converges almost surely towards $H$, when $T \rightarrow+\infty$.

The estimator $\hat{H}_{k, N, T}(X)$ is defined in a way that ensures consistency with observations of the process $X$ at two different scales. More generally, this approach can be extended to a greater number of scales [10]. The estimator of the Hurst exponent is then defined with the help of a logarithmic regression. Indeed, $-k H$ is the slope of the regression of $\log \left(M_{k, N, T}(X)\right)$ on $\log (N)$.

The property of convergence of $\check{H}_{k, N, T}($.$) and \hat{H}_{k, N, T}($.$) toward H$ holds if applied to a fBm $X$ of Hurst exponent $H$, but not if applied to $Y$, a delampertized $\mathrm{fBm}$ of underlying Hurst exponent $H$. In this latter case, such estimators are biased. The theoretical formula of the expected value of the empirical absolute moment gives a clear support to the existence of a bias. Indeed, we show, in the following theorem, that $\mathbb{E}\left[M_{k, N, T}(Y)\right]$ writes in such a way that neither $\check{H}_{k, N, T}(Y)$, nor $\hat{H}_{k, N, T}(Y)$, nor an estimator based on a regression on several scales, can be a good estimator of $H$, in the case of the delampertized $\mathrm{fBm} Y$.

Theorem 2. Let $k, N, T \in \mathbb{N}, H \in(0,1), \theta>0, X$ be a fBm of Hurst exponent $H$ and volatility $\sigma$, and $Y_{t}=\left(\mathcal{L}_{H}^{-1} X\right)_{\theta t}$. We have

$$
\mathbb{E}\left[M_{k, N, T}(X)\right]=A(\sigma, k) N^{-k H}
$$

and

$$
\mathbb{E}\left[M_{k, N, T}(Y)\right]=A(\sigma, k)\left[2-h\left(\frac{\theta}{N}\right)\right]^{k / 2},
$$

where $A(\sigma, k)=\frac{2^{k / 2} \Gamma\left(\frac{k+1}{2}\right)}{\Gamma\left(\frac{1}{2}\right)} \sigma^{k}$ and $h: x \geq 0 \mapsto 2 \cosh (H x)-\left(2 \sinh \left(\frac{x}{2}\right)\right)^{2 H}$. Moreover, we have asymptotically, when $\frac{\theta}{N} \rightarrow 0$ :

$$
\mathbb{E}\left[M_{k, N, T}(Y)\right]=A(\sigma, k) \theta^{k H} N^{-k H}+\mathcal{O}\left(\frac{\theta}{N}\right)^{k H+\min (1,2-2 H)}
$$

The consequences of Theorem 2 are the following, also summarized graphically in Figure 1 .

$\triangleright$ Low scales, that is to say higher values of $\theta / N$ : The locally perceived Hurst exponent is very low and tends toward zero, since $\mathbb{E}\left[M_{k, N, T}(Y)\right]$ decays exponentially towards $2^{k / 2} A(\sigma, k)$ when $N$ tends toward 0 .

$\triangleright$ High scales, that is to say lower values of $\theta / N$ : The locally perceived Hurst exponent becomes close to the underlying Hurst exponent as $\theta / N \rightarrow 0$.

$\triangleright$ Role of $\theta$ : This parameter changes the scale of the process and thus shifts the frontier between flattened and accurate perception of the Hurst exponent.

In other words, if one was tempted to estimate directly a Hurst exponent on the series $Y$, as if it was a $\mathrm{fBm}$, the perceived Hurst exponent would depend on the scale considered and it would in general underestimate the underlying Hurst exponent. The bias is even stronger that the resolution is low (or the scale high), which implies that $Y$ is perceived as a process with very low Hurst exponent: the mean reversion effect, the anti-persistence, or the stationarity are predominating. On the 


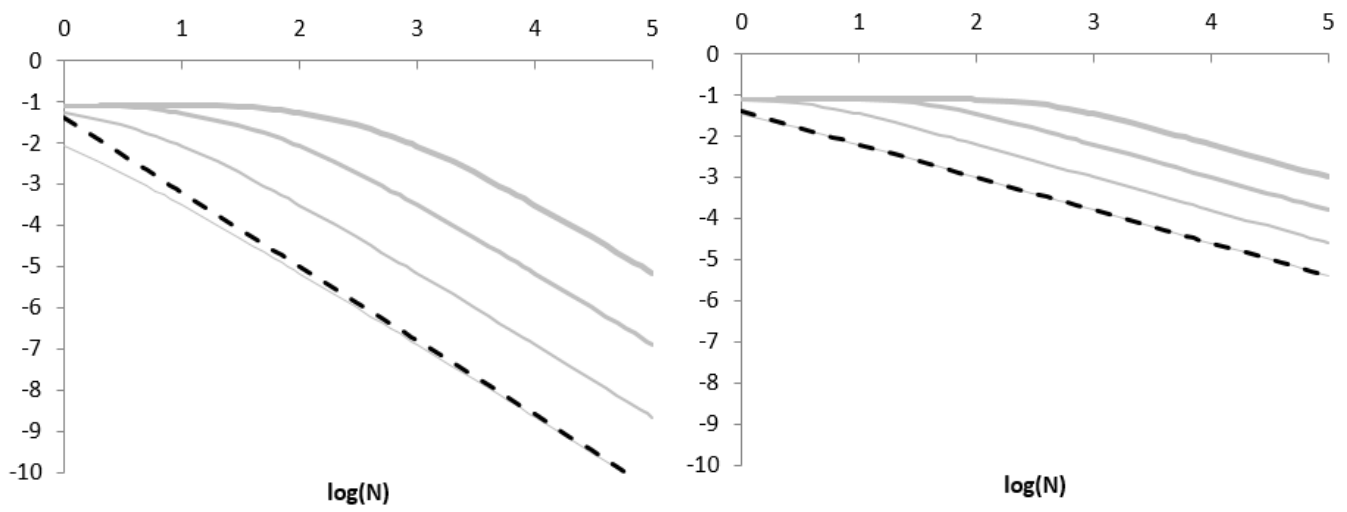

Figure 1: Value of $\log \left(\mathbb{E}\left[M_{k, N, T}(X)\right]\right) \quad$ (black) and $\log \left(\mathbb{E}\left[M_{k, N, T}(Y)\right]\right)$ (grey, for several values of $\theta$, from the thinnest to the thickest: $1,10,100,1000)$ as a function of $\log (N)$. The underlying Hurst exponent $H$ is equal to 0.9 (left) and 0.4 (right), whereas $k=2$ and $\sigma=0.2$. For a given resolution, the perceived Hurst exponent should be $-1 / k$ times the slope of the curve at this resolution.

opposite, for high resolutions (or low scales), the perception of $Y$ is consistent with $X$, the bias in the perceived Hurst exponent disappears, and the fBm feature plays a major role.

We are thus facing a stochastic process whose aspect, that is to say the Hurst statistic, depends on the resolution at which it is observed. We may perceive a Hurst exponent either close to the underlying one, or close to zero. Between these extremes, a short range of resolutions provides the statistician with a mitigated bias. The location of this transition zone depends monotonically on $\theta$. This kind of model is not disconnected from reality. For example, the series of the durations of cardiac interbeat intervals, which is expected to be stationary when the patient remains at rest, shows a crossover phenomenon: two Hurst exponents are perceived, one for small scales and the other for larger scales [30. This empirical observation could be astonishing without the theoretical justification by a delampertized $\mathrm{fBm}$.

We put forward the possibility to estimate the Hurst exponent of a fBm by taking into account a wide range of scales instead of only two. The estimated Hurst exponent is then obtained by a regression: it is $-1 / k$ times the average slope of the curve $\log (N) \mapsto \log \left(\mathbb{E}\left[M_{k, N, T}(Y)\right]\right)$, for the range of considered resolutions. Such an estimator, in the case of a delampertized fBm, thus does not help avoiding the bias. This bias will be even stronger that $\theta$ is high. In fact, fitting a linear regression, whereas the theoretical shape of $\log (N) \mapsto \log \left(\mathbb{E}\left[M_{k, N, T}(Y)\right]\right)$ is far from a line, is unreasonable.

The perceived Hurst exponent and the underlying one may differ, not only because of the inaccuracy intrinsic to statistical estimators, but mostly because of the bad specification of the model. In the literature, most estimators used are related to a perceived Hurst exponent, based on the $\mathrm{fBm}$. But for other models, in particular for a delampertized $\mathrm{fBm}$, if one follows the custom and estimates undeservedly the Hurst exponent with the standard fBm-based method, the result will be meaningless, as it will depend on the scale, and the perceived Hurst exponent may strongly underestimate the underlying one. Nevertheless, a properly estimated underlying Hurst exponent says more about the persistence of the underlying fBm than about the behaviour of its delampertized version. The interpretation of this statistic is thus challenging. 


\subsection{Interpreting the underlying Hurst exponent}

For a $\mathrm{fBm}$, the Hurst exponent depicts the persistence of the process:

$\triangleright$ if $H>1 / 2$, the process is persistent: increments are positively autocorrelated,

$\triangleright$ if $H<1 / 2$, the process is anti-persistent: increments are negatively autocorrelated,

$\triangleright$ if $H=1 / 2$, the process is a martingale: increments are not autocorrelated.

For a delampertized fBm of underlying Hurst exponent $H$, the interpretation of $H$ is different from the $\mathrm{fBm}$ case. Indeed, a positive autocorrelation is inconsistent with the stationary property of the delampertized fBm. Nevertheless, from the previous subsection, we know that this process behaves differently at low and high resolutions. When focusing on small scales, it appears indeed as a $\mathrm{fBm}$, whereas the mean reversion leads the dynamic at higher scales. Therefore, for $H>1 / 2$, we expect the autocorrelation to be positive between close increments of short duration and negative for non-overlapping increments of long duration. The following findings will confirm this intuition.

In Theorem 11. since $X_{0}=0, \operatorname{Cov}\left(X_{s}, X_{u}-X_{t}\right)$ is the covariance of non-overlapping increments of duration $s$ and $u-t$. It is therefore not surprising to find a positive covariance for $H>1 / 2$, a negative one for $H<1 / 2$, and a zero one for $H=1 / 2$. On the contrary, $Y_{0}=X_{1}$, so that $\operatorname{Cov}\left(Y_{s}, Y_{u}-Y_{t}\right)$ does not match with the covariance of increments of duration $s$ and $u-t$. This covariance of increments may in fact be positive, depending on $s, t, u, \theta$ and $H$. We show this property of $Y$ in the following proposition, restricted to the case of adjacent increments of same duration.

Proposition 1. Let $H \in(0,1), s, \theta>0, X$ be a fBm of Hurst exponent $H$ and volatility $\sigma$, and $Y_{t}=\left(\mathcal{L}_{H}^{-1} X\right)_{\theta t}$. We then have the following properties:

1. The covariance of adjacent increments of $Y$, of duration $s$, is:

$$
C(s)=\operatorname{Cov}\left(Y_{s}-Y_{0}, Y_{2 s}-Y_{s}\right)=\frac{\sigma^{2}}{2}[2 h(\theta s)-2-h(2 \theta s)]
$$

where $h: x \geq 0 \mapsto 2 \cosh (H x)-\left(2 \sinh \left(\frac{x}{2}\right)\right)^{2 H}$, with the asymptotic property:

$$
\lim _{s \rightarrow+\infty} C(s)=-\sigma^{2} .
$$

2. The correlation of adjacent increments of $Y$, of duration $s$, is:

$$
\rho(s)=\operatorname{corr}\left(Y_{s}-Y_{0}, Y_{2 s}-Y_{s}\right)=-1+\frac{2-h(2 \theta s)}{4-2 h(\theta s)},
$$

with the asymptotic property:

$$
\lim _{s \rightarrow+\infty} \rho(s)=-\frac{1}{2}
$$

The correlation of increments of same duration is displayed in Figure 2 , for various Hurst exponents. The usual interpretation of the Hurst exponent does not hold in the case of a delampertized fBm: autocorrelation is of course negative for $H<1 / 2$ but, for $H>1 / 2$, it is positive for increments of short duration and negative else.

For a delampertized fBm of parameters $H$ and $\theta$, the Hurst exponent theoretically perceived ranges between 0 and $H$, depending on the scale at which the process is observed. If $H>1 / 2$, there is a 


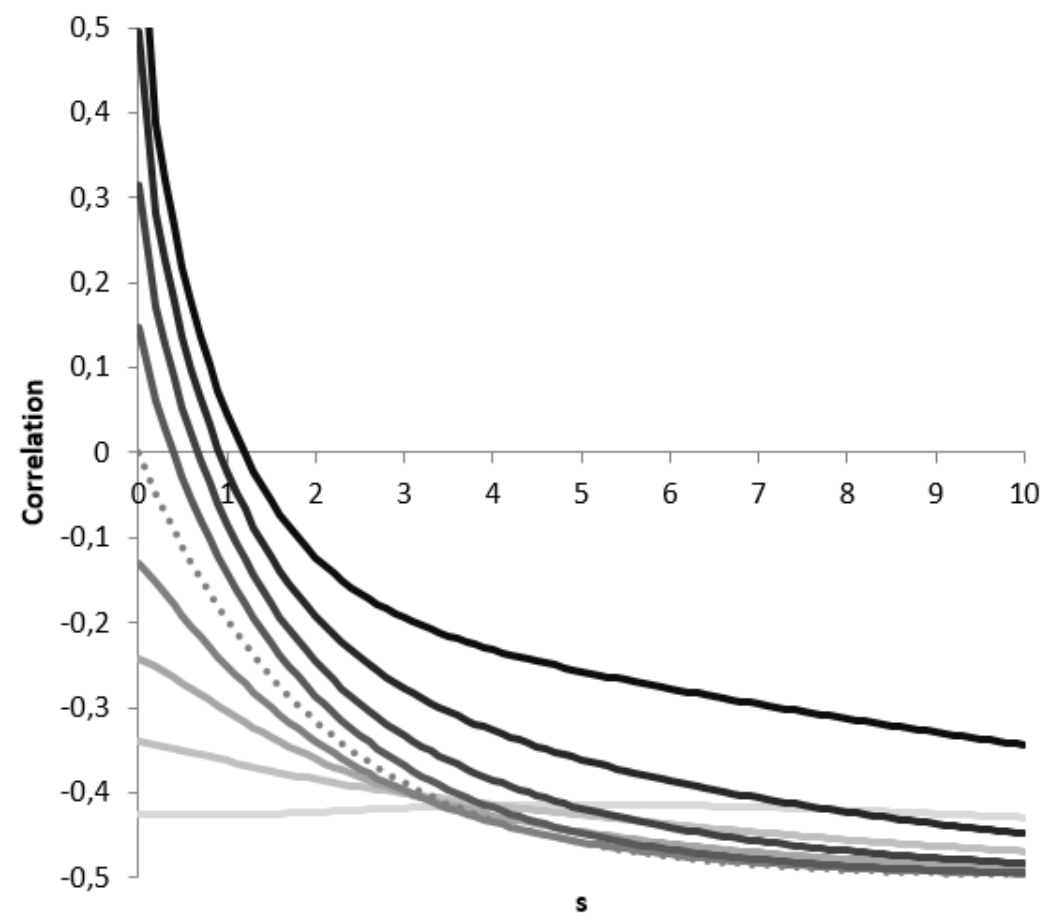

Figure 2: Correlation of adjacent increments of a delampertized $\mathrm{fBm}$ of underlying Hurst exponent $H$ equal to 0.1 (the lightest), $0.2, \ldots$, 0.9 (the darkest). The dotted curve corresponds to $H=0.5$. The parameter $\theta$ is here equal to 1 . 
scale $\eta(H, \theta)$ under which the theoretically perceived Hurst exponent is greater than $1 / 2$. We call this threshold scale the Hurst horizon. We define it as the scale $1 / N$ such that the slope of our regression corresponds to a perceived Hurst exponent of $1 / 2$ :

$$
\frac{1}{2}=-\frac{1}{k} \frac{\partial \log \left(\mathbb{E}\left[M_{k, N, T}(Y)\right]\right)}{\partial \log (N)} .
$$

Therefore, $\eta(H, \theta)$ is the solution of the following equation in $x: h(\theta x)-2=h^{\prime}(\theta x) \theta x$. Moreover, it is straightforward that $\eta(H, \theta)=\eta(H, 1) / \theta$ : when the time scale of the series is contracted by a factor $\theta$, the Hurst horizon is contracted as well.

Intuitively, the Hurst horizon provides us with a rough idea of the scale at which increments are positively correlated. It is only an approximation. For instance, if we consider two adjacent increments of same duration, the maximum duration $s$ for which the pair of increments is positively correlated is below $\eta(H, \theta)$, whatever $H$ and $\theta$, whereas the size of the interval containing both increments, $2 s$, is above $\eta(H, \theta)$, as exposed in Figure 3. Nevertheless, $s$ and $\eta(H, \theta)$ evolve concomitantly, regarding $H$, what confirms the intuition behind the Hurst horizon.

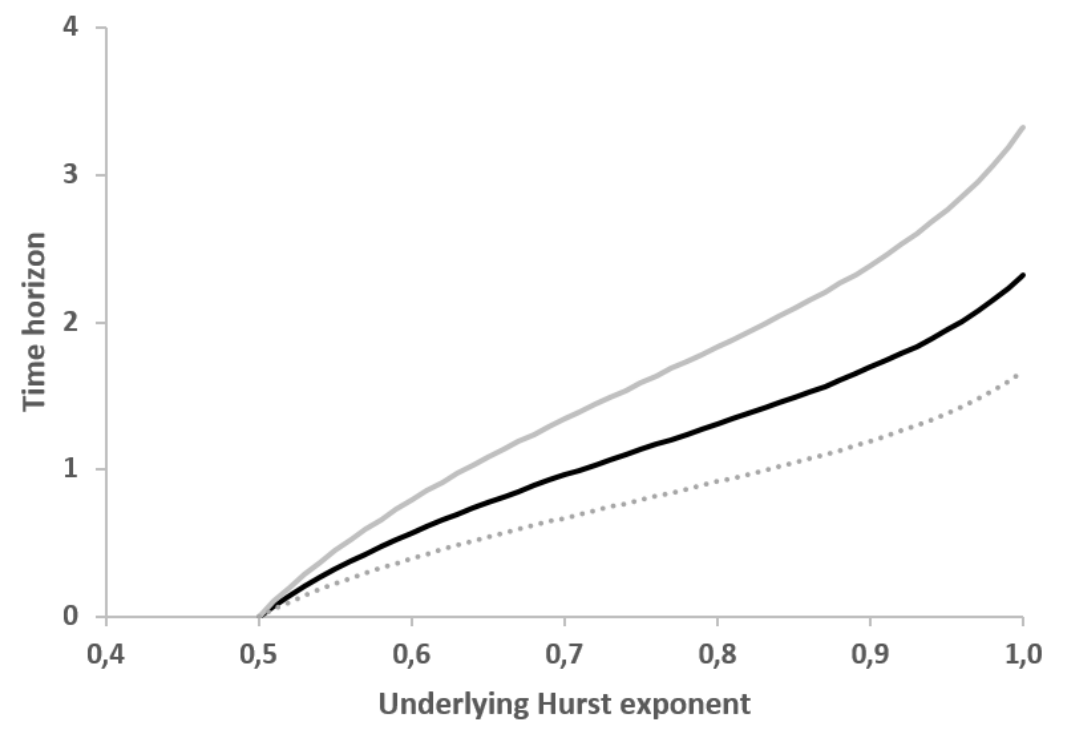

Figure 3: Hurst horizon (black), maximal duration $s$ such that an increment of duration s of a delampertized $\mathrm{fBm}$ is positively correlated to the adjacent increment of same duration (grey dots), duration of the interval containing these two increments (grey line), as a function of the underlying Hurst exponent. The grey dotted line corresponds in fact to the intercept of the various curves represented in Figure 2 A linear regression with a $\mathrm{R}^{2}$ equal to 0.9997 provides us with the relation $s=0.7052 \times \eta(H, \theta)$. The parameter $\theta$ is here equal to 1 .

Even though a delampertized fBm may have positively correlated increments, it does not hold longrange dependence, due to the exponential decay of the autocorrelation, as stated in the following proposition.

Proposition 2. Let $H \in(0,1), s, \theta>0, d \geq 0, X$ be a fBm of Hurst exponent $H$, and $Y_{t}=$ $\left(\mathcal{L}_{H}^{-1} X\right)_{\theta t}$. The correlation of adjacent increments of $Y$, of duration $s$, separated by an interval of 
duration $(d-1) s$ is then:

$$
\rho(s, d)=\operatorname{corr}\left(Y_{s}-Y_{0}, Y_{(d+1) s}-Y_{d s}\right)=\frac{2 h(d \theta s)-h((d-1) \theta s)-h((d+1) \theta s)}{4-2 h(\theta s)},
$$

where $h: x \geq 0 \mapsto 2 \cosh (H x)-\left(2 \sinh \left(\frac{x}{2}\right)\right)^{2 H}$, with the asymptotic property:

$$
\rho(s, d) \stackrel{d \rightarrow+\infty}{\sim} \begin{cases}\left(\frac{1-\cosh (H \theta s)}{2-h(\theta s)}\right) e^{-d H \theta s} & \text { if } H \leq \frac{1}{2} \\ 2 H\left(\frac{1-\cosh ((1-H) \theta s)}{2-h(\theta s)}\right) e^{-d(1-H) \theta s} & \text { else. }\end{cases}
$$

This is consistent with the fact that this kind of process looks like a fBm for small scales and like a mean-reverting process at higher scales. On the opposite, a fBm has either only negatively correlated increments and no long-range dependence, or only positively correlated increments and long-range dependence, depending on the value of its Hurst exponent. Indeed the correlation between two increments of a $\mathrm{fBm}$, of duration $s$, and separated by an interval of duration $(d-1) s$, is $\left((d+1)^{2 H}-2 d^{2 H}-(d-1)^{2 H}\right) / 2$.

Provided that $\theta s$ is small enough, we also observe positive correlations for $H>1 / 2$, at least for increments close in time. However, for larger values of $\theta s$, the mean-reverting aspect is predominant and autocorrelations are always negative, whatever the value of $H$ and of $d$. This is illustrated by Figure 4

It is worth noting that the fOU process, despite its stationarity, holds a long memory if its underlying Hurst exponent is above $1 / 2$ [ 6 . It is one of the main differences between the fOU approach and the delampertized fBm. This difference can lead to the preference of one of the two models, depending on the existence or not of a long-memory feature in the data. In particular, we show in the next section that the FX rates series we are working with do not hold long memory, whereas their underlying Hurst exponent is above 1/2. This makes the use of the delampertized fBm more relevant than the fOU process.

\section{Application to high-frequency FX rates}

We propose an application to financial time series of the delampertized fBm model, in which the perceived Hurst exponent may depend monotonically on the scale. In particular, we are interested in foreign exchange rates. Many studies of their predictability have already been published. Among them, we can cite techniques based on fractional dynamics and Hurst exponents [15, 13, on multiscale decomposition [2, 18, or on chaos theory [12, 17, 13. In the latter case, one of the challenges consists in determining the Lyapunov horizon, under which forecasts are supposed reliable. In the case of fractional dynamics, the reliability of the forecasts usually only relies on the value of the estimated Hurst exponent: if it is below 0.5, it is better to avoid any forecast, whereas greater values of Hurst exponents should make the forecasts reliable for a quite long horizon, because of a long-memory feature. The present paper brings two remarks concerning this belief:

$\triangleright$ The perceived Hurst exponent may strongly underestimate the underlying one, so that time series reputed to be unpredictable, because of a low Hurst exponent, may in fact be a transform of a fBm with a Hurst exponent above 0.5. In other words, asserting that these series are unpredictable may be wrong.

$\triangleright$ The inverse Lamperti transform of a $\mathrm{fBm}$ is not a long-memory process. We introduced the Hurst horizon, beyond which forecasts should be unreliable because increments become 

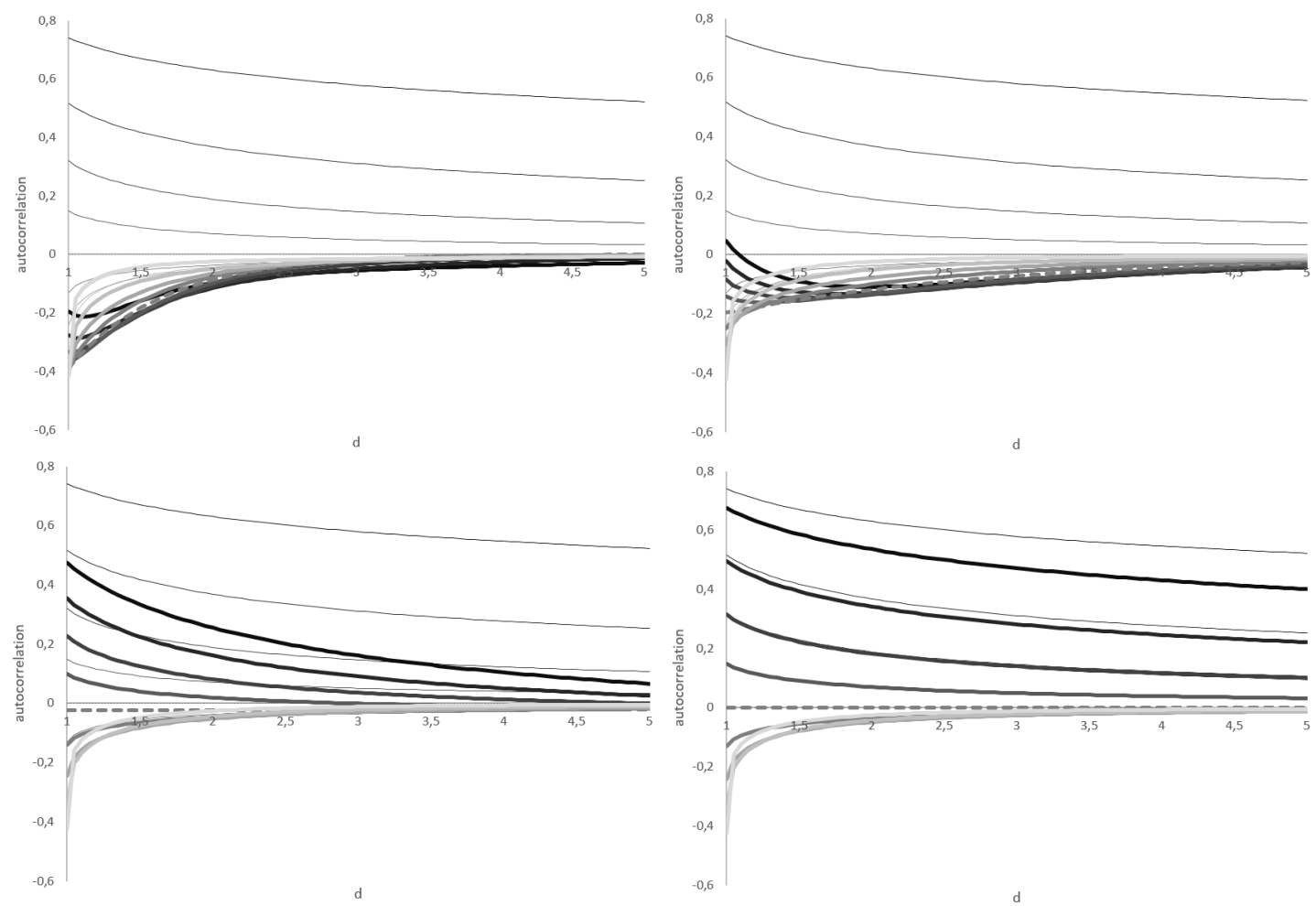

Figure 4: Correlation of increments of duration 1 separated by an interval of duration $(d-1)$ for a $\mathrm{fBm}$ (thin lines) and a delampertized fBm (thick lines) of underlying Hurst exponent $H$ equal to 0.1 (the lightest), $0.2, \ldots, 0.9$ (the darkest). The dotted curves correspond to $H=0.5$. The parameter $\theta$ is here equal to 3 (top left), 1 (top right), 0.1 (bottom left) or 0.001 (bottom right). 
negatively correlated. Therefore, whatever the estimated Hurst exponent, predictions might be feasible for small scales up to the Hurst horizon.

Our application is based on nine FX rates versus Euro: the British pound (GBP), the Swiss franc (CHF), the Swedish krona (SEK), the United States dollar (USD), the Canadian dollar (CAD), the Australian dollar (AUD), the Japanese yen (JPY), the Chinese yuan (CNY), and the Singapore dollar (SGD). The series begin the 7th March 2016 and finish the 7th September 2016, with a time step of 15 minutes while the market is open. The exchange rate considered is the average between the highest and the lowest rate in the 15-minute time interval. For each exchange rate, we thus have roughly 12,930 observations. The CNY/EUR rate is an exception, with only 7,422 observations, because of discontinuous trading.

\subsection{Relevance of the delampertized fBm}

In order to apply the delampertized fBm to FX rates, we have to justify that this model is relevant. In particular, two features are of importance: the stationarity of the process and the presence or not of long memory. For its part, the fractional nature of FX rates series is now well established [35, 5. 15, 36 and makes it possible to generalize dynamics based on the standard Brownian motion.

For the stationarity, we performed an augmented Dickey-Fuller (ADF) test, with trend and intercept. The null hypothesis of this test is the presence of a unit root and therefore of non-stationarity. We see in Table 1, that this hypothesis is rejected for two pairs: SEK/EUR and CNY/EUR. This proves the significance of the stationarity of these two series, even though, for the seven other series, the stationarity is not proved statistically. Using a stationary process, like the delampertized $\mathrm{fBm}$, to model FX rates is thus relevant, at least for some pairs of currencies.

Among fractional stationary processes, we can cite the delampertized fBm and the fOU process. The preference to one or the other process should be lead by the presence or absence or long memory. The delampertized $\mathrm{fBm}$ is not a long-memory process, since its autocorrelation function is an exponential function [6]. On the contrary, the fOU process with a Hurst exponent higher than 0.5 has a long memory, with a power function for its autocorrelation [6. As a consequence, we computed the empirical autocorrelation $R(l)$ for each FX rate series ${ }^{2}$ with a lag $l$ ranging from 1 to 1,000 time steps ${ }^{3}$ We want to determine which of the two following models best fits the empirical autocorrelation:

$\triangleright \ln (R(l))=-\beta l+\varepsilon_{l}$, which corresponds to an exponential decay of the autocorrelation,

$\triangleright \ln (R(l))=-\beta \ln (l)+\varepsilon_{l}$, which corresponds to a power decay of the autocorrelation.

In both models, $\beta>0$ and $\varepsilon_{l}$ are Gaussian i.i.d. variables. We make two linear regressions of $\ln (R(l))$ on either $l$ or $\ln (l)$, for $l=1,2, \ldots, 1,000$, with a maximum likelihood estimation. We determine the information loss induced by each model, namely the Akaike information criterion (AIC), which, in our framework of Gaussian residuals, is equal to 2 minus 2 times the sum of squared errors of the regression 4 The lower is the AIC, the more suitable is the model. As we can see in Table 1 all the pairs have a lower AIC for the exponential model of autocorrelation decay than for the power model. This means that the exponential decay is more likely than the power one. The extent to which the exponential decay is more likely is quantified by the relative likelihood,

\footnotetext{
${ }^{2}$ We worked directly with the rate series, not with the series of the variations of the rate.

${ }^{3}$ In other words, the lag is between 15 minutes and more than 10 days.

${ }^{4}$ As the two models have the same number of parameters, the BIC would lead to the same conclusions as the AIC.
} 
$\exp \left(\frac{1}{2}\left[A I C_{\text {exp }}-A I C_{\text {pow }}\right]\right)$. For example, for SGD/EUR, the relative likelihood is $2.95 \times 10^{-4}$, what means that the power decay of the autocorrelation function is $2.95 \times 10^{-4}$ as likely as the exponential decay.

\begin{tabular}{l||c||c|c|c}
\hline \hline & $\begin{array}{c}\text { ADF } \\
\text { tau-stat. }\end{array}$ & $\begin{array}{c}\text { AIC } \\
\text { exp. decay }\end{array}$ & $\begin{array}{c}\text { AIC } \\
\text { power decay }\end{array}$ & $\begin{array}{c}\text { Relative } \\
\text { likelihood }\end{array}$ \\
\hline \hline GBP/EUR & -1.42 & 2.03 & 10.48 & $1.46 \times 10^{-2}$ \\
CHF/EUR & -2.57 & 2.37 & 32.77 & $2.51 \times 10^{-7}$ \\
SEK/EUR & $-3.20^{\star}$ & 2.26 & 5.50 & $1.99 \times 10^{-1}$ \\
\hline USD/EUR & -3.12 & 8.86 & 99.38 & $2.20 \times 10^{-20}$ \\
CAD/EUR & -2.91 & 9.12 & 300.32 & $5.84 \times 10^{-64}$ \\
AUD/EUR & -1.68 & 5.22 & 52.03 & $6.83 \times 10^{-11}$ \\
\hline JPY/EUR & -2.36 & 2.08 & 4.39 & $3.15 \times 10^{-1}$ \\
CNY/EUR & $-3.99^{\star \star \star}$ & 12.63 & 251.27 & $1.52 \times 10^{-52}$ \\
SGD/EUR & -2.37 & 2.55 & 18.81 & $2.95 \times 10^{-4}$ \\
\hline \hline
\end{tabular}

Table 1: Statistic of the stationarity test with p-value $\leq 0.1\left(^{\star}\right)$ and $\left.\leq 0.01{ }^{\star \star \star}\right)$. AIC for an exponential and power decay, and the corresponding relative likelihood of the power decay with regard to the exponential decay.

The long-memory feature is thus clearly refuted in our dataset, what is not always the case in the literature for other samples of FX rates [7, 28. However, other studies confirm our findings with a twofold behaviour of the series, and without long memory at low frequency [5. Thus, we are inclined to work with the delampertized fBm rather than with the fOU process.

\subsection{Results}

All our estimations of Hurst exponents are based on the absolute-moment estimator of order 25 For each FX rate, we estimate:

$\triangleright$ the perceived Hurst exponent, calculated as half the slope of $\log \left(M_{2,2^{-i} / \tau, T}(X)\right)$ on $\log \left(\tau 2^{i}\right)$, for $i$ ranging from 0 to 11 and $\tau$ equal to the minimum time step, namely 15 minutes (therefore $\tau 2^{i}$ is between 15 minutes and 21 days),

$\triangleright$ the underlying Hurst exponent, estimated as the Hurst exponent at the finest scale (the limit of half the slope mentioned above when $i \rightarrow 0$ ),

$\triangleright$ the empirical Hurst horizon, estimated as the smallest scale for which the above-mentioned half slope reaches 0.5 (we also call thereafter local Hurst exponent the perceived Hurst exponent for a given isolated scale). It is linearly interpolated between $\tau 2^{i-1}$ and $\tau 2^{i}$, where $\tau 2^{i}$ is the first scale with a Hurst exponent below 0.5.

Results are gathered in Table 2. The standard regression-based estimation technique leads to a Hurst exponent lower than 0.5, except for GBP/EUR. This means that the perceived Hurst exponent almost always encourages not to make any forecast. Nevertheless, we see in two examples, Figures 5 and 6 , that the scaling rule varies with the scale and therefore that a global Hurst exponent is not enough to depict how the series behaves across scales. Indeed, for small scales, we found

\footnotetext{
${ }^{5}$ In Figures 5,6 and 7 , we also display estimations for orders 1, 3, and 4, which confirm the findings related to order 2 .
} 
systematically a local Hurst exponent above 0.5 , whereas lowering the resolution leads to lower Hurst exponents.

\begin{tabular}{l|c|c|c}
\hline \hline & $\begin{array}{c}\text { Perceived } \\
\text { Hurst exponent }\end{array}$ & $\begin{array}{c}\text { Underlying } \\
\text { Hurst exponent }\end{array}$ & $\begin{array}{c}\text { Empirical } \\
\text { Hurst horizon }\end{array}$ \\
\hline \hline GBP/EUR & 0.542 & 0.680 & $7 \mathrm{~d} 15 \mathrm{~h} 24 \mathrm{~m}$ \\
CHF/EUR & 0.448 & 0.652 & $2 \mathrm{~h} 20 \mathrm{~m}$ \\
SEK/EUR & 0.449 & 0.668 & $1 \mathrm{~h} 33 \mathrm{~m}$ \\
\hline USD/EUR & 0.466 & 0.694 & $4 \mathrm{~h} 53 \mathrm{~m}$ \\
CAD/EUR & 0.457 & 0.668 & $4 \mathrm{~h} 19 \mathrm{~m}$ \\
AUD/EUR & 0.498 & 0.669 & $2 \mathrm{~h} 16 \mathrm{~m}$ \\
\hline JPY/EUR & 0.493 & 0.703 & $5 \mathrm{~h} 28 \mathrm{~m}$ \\
CNY/EUR & 0.418 & 0.604 & $3 \mathrm{~h} 1 \mathrm{~m}$ \\
SGD/EUR & 0.456 & 0.645 & $1 \mathrm{~h} 49 \mathrm{~m}$ \\
\hline \hline
\end{tabular}

Table 2: Estimations of Hurst exponents and of Hurst horizons (in days, hours, and minutes). The perceived Hurst exponent is based on a regression for scales $2^{i} \times 15$ minutes, for $i$ ranging from 0 to 11 .

This finding is also confirmed graphically in Figures 5 and 6 for other orders of absolute moments. For $k=2$, the absence of linearity between the log absolute moment and the log resolution is not striking at first glance, though it is real as does certify the slight concavity of the curve compared to the linear regression, or the computation of the local Hurst exponent. But for higher orders, the variation of the slope with the scale is obvious. The time series of foreign exchange rates thus cannot be a simple fBm.

This scaling law is in fact typical of a process acting as a fBm of high Hurst exponent at low scales, and as a mean-reverting process at higher scales, exactly like the delampertized fBm. Such a twofold behaviour also appears graphically in Figures 5 and 6 , with the historical FX rate at two scales. At a low resolution, the series seems unpredictable, made of peaks and mean reversions, whereas, at the scale of a day, clear patterns, based on a positive autocorrelation, make predictions feasible.

In this framework, we also display in Table 2 the underlying Hurst exponent and the empirical Hurst horizon. The underlying Hurst exponent is much higher than the perceived one, always above 0.6. This leads to the counter-intuitive fact that reliable predictions could be made for all these FX rates, even though the perceived Hurst exponent is lower than 0.5. Nevertheless, the estimated horizon of reliability of these predictions is quite short, between 1 hour 33 minutes (SEK/EUR) and 5 hours 28 minutes (JPY/EUR).

The case of GBP/EUR is different, with a long Hurst horizon of more than a week. We explain this by the fact that it is the only pair studied with a perceived Hurst exponent above 0.5. This high value of the perceived Hurst exponent may suggest that there is no mean reversion in this series and, thus, that the delampertized fBm is not relevant for GBP/EUR. In fact, this perceived Hurst exponent is an average of local Hurst exponents on several scales. Contrary to many FX rates, the scale above which the local Hurst exponent is below 0.5 is very high, as we can see in Table 2 and as the local Hurst exponents displayed in Figure 7 confirm. Therefore, with our sample limited to 6 months, our perceived Hurst exponent of GBP/EUR is an average of many Hurst exponents above 0.5 and of only some below. This means that, if we increased the set of scales considered or if we overweighted high scales, we would get a perceived Hurst exponent lower than 0.5. Mean reversion thus exists for GBP/EUR too, but at a much higher scale than for other series.

In Figures 5 , 6, and 7, we observe a peak in each series of rates in June 2016. These peaks 

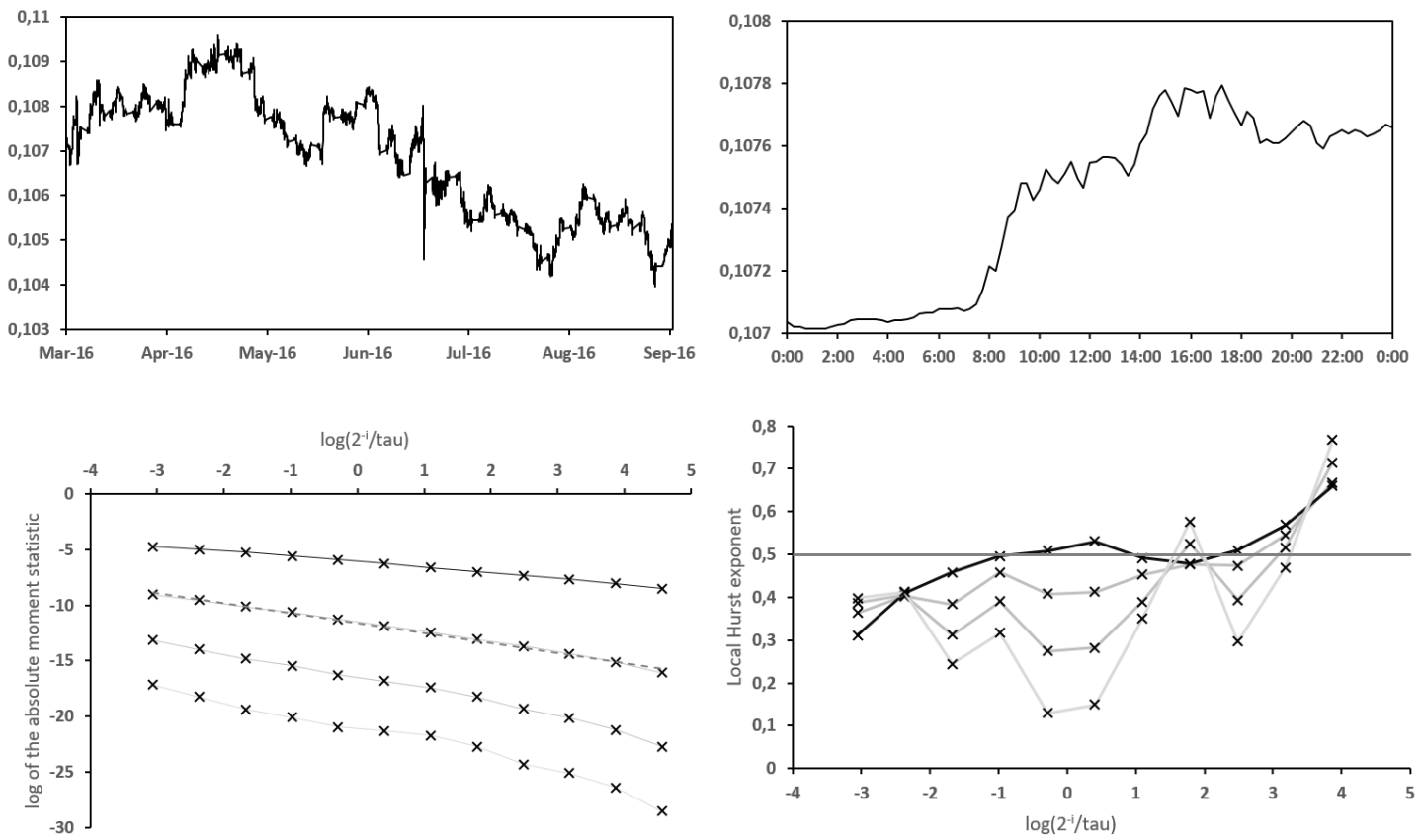

Figure 5: SEK/EUR rate of all our sample (top left) and with a focus on the 9th March 2016 (top right). Bottom left: log empirical absolute moment on the log of the inverse time step, for moments of order $k=1$ (black) to $k=4$ (lightest grey); a linear regression is added as a dotted line for $k=2$. Bottom right: corresponding local Hurst exponents, defined, for the scale $\log \left(2^{-i} / \tau\right)$, as $-1 / k$ times the slope of the bottom-left curve between scales $\log \left(2^{-i} / \tau\right)$ and $\log \left(2^{-(i-1)} / \tau\right)$. 

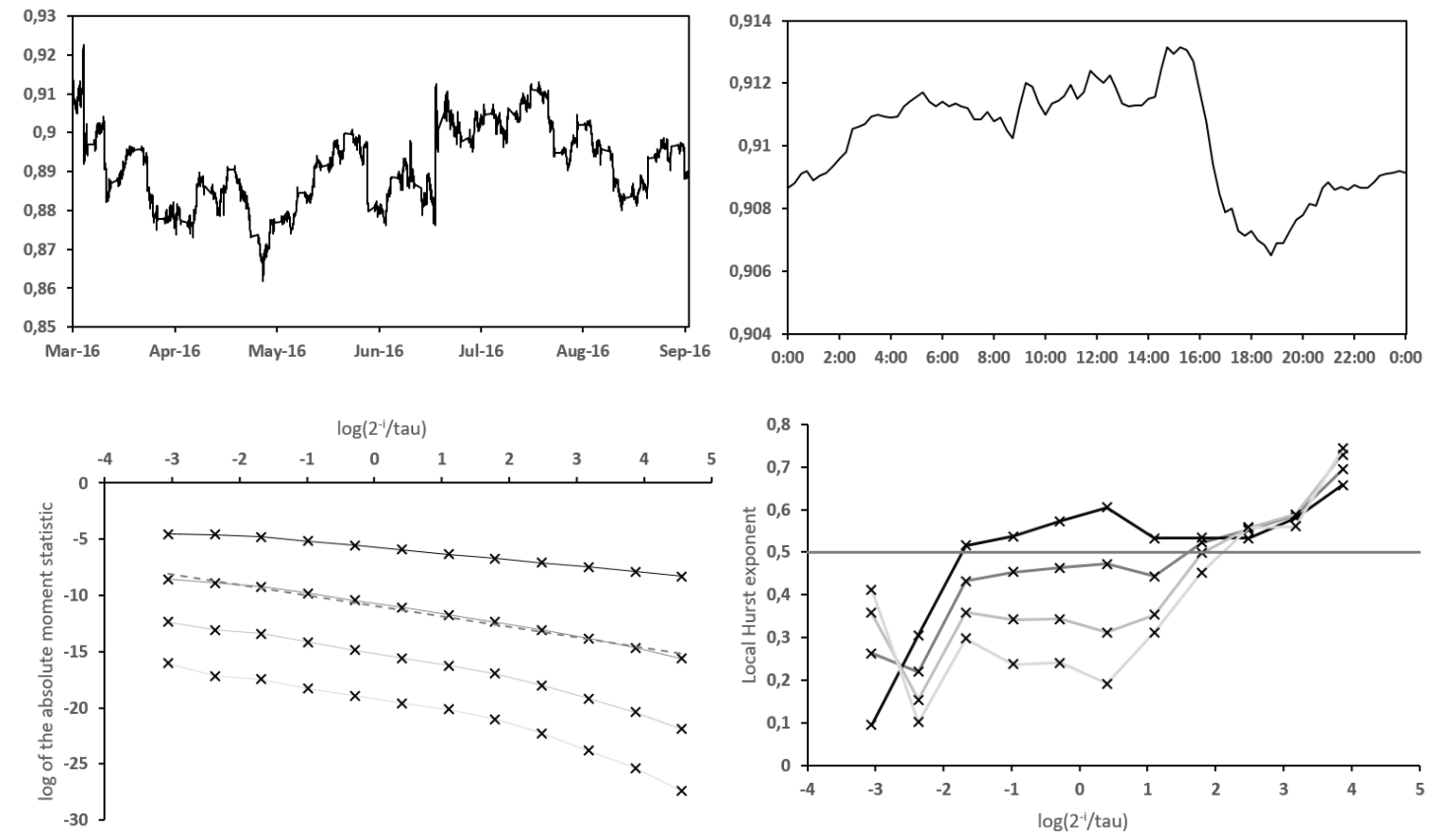

Figure 6: USD/EUR rate of all our sample (top left) and with a focus on the 9th March 2016 (top right). Bottom left: log empirical absolute moment on the log of the inverse time step, for moments of order $k=1$ (black) to $k=4$ (lightest grey); a linear regression is added as a dotted line for $k=2$. Bottom right: corresponding local Hurst exponents, defined, for the scale $\log \left(2^{-i} / \tau\right)$, as $-1 / k$ times the slope of the bottom-left curve between scales $\log \left(2^{-i} / \tau\right)$ and $\log \left(2^{-(i-1)} / \tau\right)$. 

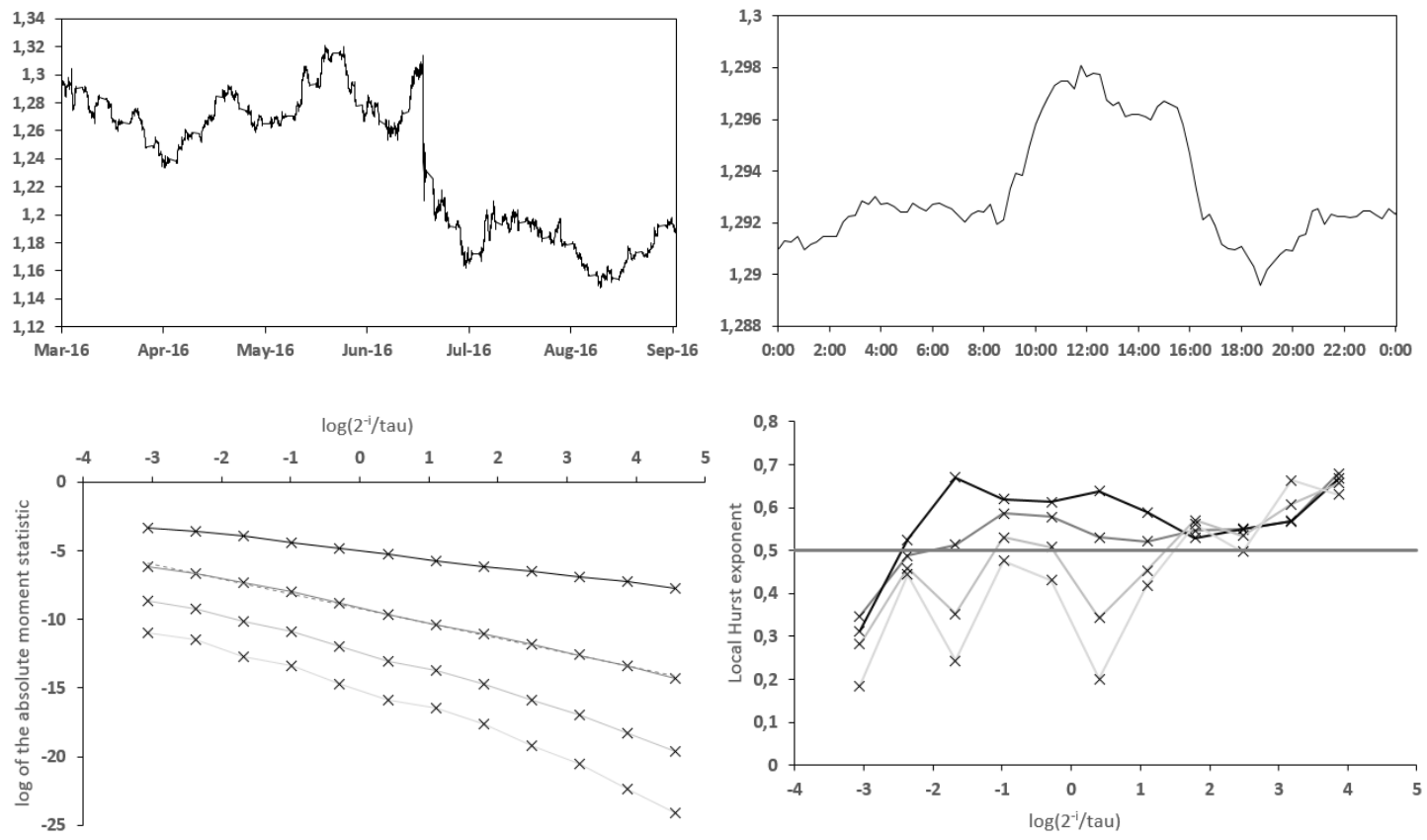

Figure 7: GBP/EUR rate of all our sample (top left) and with a focus on the 9th March 2016 (top right). Bottom left: log empirical absolute moment on the log of the inverse time step, for moments of order $k=1$ (black) to $k=4$ (lightest grey); a linear regression is added as a dotted line for $k=2$. Bottom right: corresponding local Hurst exponents, defined, for the scale $\log \left(2^{-i} / \tau\right)$, as $-1 / k$ times the slope of the bottom-left curve between scales $\log \left(2^{-i} / \tau\right)$ and $\log \left(2^{-(i-1)} / \tau\right)$. 
correspond to a market reaction after the Brexit referendum of the 23rd June, 2016. If we stop the sample on the 23rd June, just before the publication of the outcome of the referendum, we get perceived and underlying Hurst exponents very close to those estimated on the full sample, as one can see in Table 3. In particular, underlying Hurst exponents are still all above 0.5. Perceived ones are still below 0.5, except for GBP/EUR and for AUD/EUR. However, for this latter pair, the perceived Hurst exponent had not changed much ( 0.503 for the partial sample versus 0.498 for the full sample). The magnitude of the empirical Hurst horizon remains the same for all the pairs, except for CAD/EUR and JPY/EUR. Indeed, in these cases, the horizon is increased by a few hours. However, the global picture remains the same for all the series, with mean reversion at high scales, and persistence at lower scales.

\begin{tabular}{l|c|c|c}
\hline \hline & $\begin{array}{c}\text { Perceived } \\
\text { Hurst exponent }\end{array}$ & $\begin{array}{c}\text { Underlying } \\
\text { Hurst exponent }\end{array}$ & $\begin{array}{c}\text { Empirical } \\
\text { Hurst horizon }\end{array}$ \\
\hline \hline GBP/EUR & 0.516 & 0.668 & $7 \mathrm{~d} 14 \mathrm{~h} 15 \mathrm{~m}$ \\
$\mathrm{CHF} / \mathrm{EUR}$ & 0.482 & 0.653 & $1 \mathrm{~h} 23 \mathrm{~m}$ \\
$\mathrm{SEK} / \mathrm{EUR}$ & 0.454 & 0.647 & $1 \mathrm{~h} 28 \mathrm{~m}$ \\
\hline $\mathrm{USD} / \mathrm{EUR}$ & 0.471 & 0.704 & $3 \mathrm{~h} 57 \mathrm{~m}$ \\
$\mathrm{CAD} / \mathrm{EUR}$ & 0.478 & 0.677 & $12 \mathrm{~h} 48 \mathrm{~m}$ \\
$\mathrm{AUD} / \mathrm{EUR}$ & 0.503 & 0.682 & $2 \mathrm{~h} 18 \mathrm{~m}$ \\
\hline JPY/EUR & 0.486 & 0.706 & $23 \mathrm{~h} 44 \mathrm{~m}$ \\
$\mathrm{CNY} / \mathrm{EUR}$ & 0.442 & 0.588 & $1 \mathrm{~h} 42 \mathrm{~m}$ \\
$\mathrm{SGD} / \mathrm{EUR}$ & 0.439 & 0.662 & $1 \mathrm{~h} 43 \mathrm{~m}$ \\
\hline \hline
\end{tabular}

Table 3: Estimations, on a partial sample, of Hurst exponents and of Hurst horizon (in days, hours, and minutes). The perceived Hurst exponent is based on a regression for scales $2^{i} \times 15$ minutes, for $i$ ranging from 0 to 11 . The sample stops the 23rd June, 2016.

Our study thus confirms that the delampertized $\mathrm{fBm}$ is relevant for modelling FX rates, with or without Brexit referendum. This result is complementary to a recent analysis which showed that the Hurst exponent of GBP/USD was higher by the Brexit referendum [36]. Indeed, a peak spread on several hours is characterized by a high autocorrelation of high-frequency increments. In our work, we show that, at a higher scale, the effect of the Brexit referendum on the persistence of FX rates is negligible.

\section{Conclusion}

In this paper, we studied the inverse Lamperti transform of a fBm. This process looks like a fBm at small scales and like a mean-reverting process at higher scales. Thanks to this transform, the fBm gains stationarity but loses its long memory in the same time.

Therefore, the usual analysis of the time series with a global Hurst exponent is flawed. In this framework, we have to distinguish the underlying Hurst exponent, which is the Hurst exponent of the fBm before it is transformed, and the perceived Hurst exponent, directly estimated on the delampertized fBm. The latter Hurst exponent is scale-dependent. The perceived Hurst exponent, estimated by the usual linear regression of the log absolute moments on the log scales, provides us with an underestimation of the underlying Hurst exponent.

We have thus several notions of Hurst exponent. A natural question is: on which of these Hurst exponents should we base the usual analysis of persistence of the series? Unfortunately, we can 
interpret neither the underlying Hurst exponent, nor the perceived one, as the usual persistence indicator. We simply state that a delampertized fBm of underlying Hurst exponent above $1 / 2$ is persistent up to a given scale, called the Hurst horizon. This incites us to believe that, in this framework, we could build reliable predictions in the limit of this Hurst horizon. The way we may make such predictions is the purpose of a future paper [16].

This model seems well suited to financial data. We found underlying Hurst exponents higher than $1 / 2$ on series of FX rates, whereas the standard analysis provided a perceived Hurst exponent lower than $1 / 2$. In other words, without this framework, we would have limited our analysis to the perceived Hurst exponent. We would thus have concluded that predictions are unreliable, because of a lack of persistence in the time series. On the contrary, with our new contribution, we can assert that predictions at small scales are relevant.

This is consistent with the findings of Bouchaud et alii in a recent paper [3, in which they show empirically that returns are predictable at small scales and not at higher scales, at least on their dataset, which consists in daily prices of commodities, stocks, and currencies. In addition, they define a model to describe this effect: an Ornstein-Uhlenbeck process with a positively autocorrelated noise. The rationale of this model is close to ours, but an important strength of our approach is that it directly relates to the Hurst literature, which is massive in econophysics.

\section{A Proof of Theorem 1}

Proof. $\quad$ 1. Thanks to equation (1), we can write:

$$
\begin{aligned}
\operatorname{Cov}\left(X_{s}, X_{u}-X_{t}\right) & =\mathbb{E}\left[X_{s} X_{u}\right]-\mathbb{E}\left[X_{s} X_{t}\right] \\
& =\frac{\sigma^{2}}{2}\left(u^{2 H}-t^{2 H}-(u-s)^{2 H}+(t-s)^{2 H}\right) .
\end{aligned}
$$

Then, three cases arise:

$\triangleright$ If $H=1 / 2$, then equation (3) immediately leads to $\operatorname{Cov}\left(X_{s}, X_{u}-X_{t}\right)=0$.

$\triangleright$ If $H>1 / 2$, then the function $\kappa: t \in[s, u) \mapsto 2 \operatorname{Cov}\left(X_{s}, X_{u}-X_{t}\right) / \sigma^{2}$ is differentiable with a derivative equal to $\kappa^{\prime}(t)=2 H\left((t-s)^{2 H-1}-t^{2 H-1}\right)$, which is equal to zero if and only if $s=0$, what is not allowed by the assumptions of the theorem. The function $\kappa$ is thus strictly decreasing. Moreover, $\lim _{t \rightarrow u} \kappa(t)=0$. Therefore, whatever $t \in[s, u), \kappa(t)$ is strictly positive. Finally, $\operatorname{Cov}\left(X_{s}, X_{u}-X_{t}\right)>0$.

$\triangleright$ If $H<1 / 2$, symmetrically to the case $H>1 / 2$, the same function $\kappa$ is strictly increasing with the same limit when $t$ tends toward $u$. Therefore, $\kappa$ is strictly negative because $2 H-1<0$ and $\operatorname{Cov}\left(X_{s}, X_{u}-X_{t}\right)<0$.

2. Thanks to equation (1), we can write:

$$
\begin{aligned}
& \operatorname{Cov}\left(Y_{s}, Y_{u}-Y_{t}\right) \\
= & e^{-\theta H(s+u)} \mathbb{E}\left[X_{\exp (\theta s)} X_{\exp (\theta u)}\right]-e^{-\theta H(s+t)} \mathbb{E}\left[X_{\exp (\theta s)} X_{\exp (\theta t)}\right] \\
= & \frac{\sigma^{2}}{2}\left[e^{-\theta H(s+u)}\left(e^{2 \theta H s}+e^{2 \theta H u}-\left(e^{\theta u}-e^{\theta s}\right)^{2 H}\right)\right. \\
& \left.-e^{-\theta H(s+t)}\left(e^{2 \theta H s}+e^{2 \theta H t}-\left(e^{\theta t}-e^{\theta s}\right)^{2 H}\right)\right] \\
= & \sigma^{2}\left[\cosh (\theta H(u-s))-\cosh (\theta H(t-s))-2^{2 H-1}\left(\sinh \left(\frac{\theta(u-s)}{2}\right)\right)^{2 H}+2^{2 H-1}\left(\sinh \left(\frac{\theta(t-s)}{2}\right)\right)^{2 H}\right] \\
= & \frac{\sigma^{2}}{2}[h(\theta(u-s))-h(\theta(t-s))] .
\end{aligned}
$$

It is strictly negative since both $\theta(u-s)>\theta(t-s)$ and $h$ is a strictly decreasing function. 
Indeed, $h(x)$ also writes $e^{H x}\left[1+e^{-2 H x}-\left(1-e^{-x}\right)^{2 H}\right]$ and, for $x>0$ and $H \in(0,1)$ :

$$
\begin{aligned}
h^{\prime}(x) & =H e^{H x}\left\{\left[1+e^{-2 H x}-\left(1-e^{-x}\right)^{2 H}\right]+\left[-2 e^{-2 H x}-2 e^{-x}\left(1-e^{-x}\right)^{2 H-1}\right]\right\} \\
& =H e^{H x}\left[1-e^{-2 H x}-\left(1+e^{-x}\right)\left(1-e^{-x}\right)^{2 H-1}\right] \\
& \leq H e^{H x}\left[1-e^{-2 H x}-\left(1+e^{-x}\right)\left(1-e^{-x}\right)\right] \\
& \leq H e^{H x}\left[e^{-2 x}-e^{-2 H x}\right] \\
& <0,
\end{aligned}
$$

in which the first inequality is quite straightforward: let $y=1-e^{-x} \in(0,1)$; if $H \leq 1 / 2$ then $2 H-1 \leq 0$ and $y^{2 H-1} \geq 1 \geq y$; if $H>1 / 2$ then $2 H-1 \in(0,1)$ and $y^{2 H-1} \geq y$.

\section{B Proof of Theorem 2}

Proof. First, for a Gaussian random variable $Z$ of mean 0 and variance 1, we have:

$$
\mathbb{E}\left[|Z|^{k}\right]=\frac{2^{k / 2} \Gamma\left(\frac{k+1}{2}\right)}{\Gamma\left(\frac{1}{2}\right)} .
$$

$\triangleright$ Therefore, since, for all $t \in \mathbb{R},\left(X_{t}-X_{t-1 / N}\right) /\left(\sigma N^{-H}\right)$ is a Gaussian random variable of mean 0 and variance 1 , we get:

$$
\mathbb{E}\left[\left|X_{t}-X_{t-1 / N}\right|^{k}\right]=\frac{2^{k / 2} \Gamma\left(\frac{k+1}{2}\right)}{\Gamma\left(\frac{1}{2}\right)} \sigma^{k} N^{-k H} .
$$

And, by linearity, the same result holds for $\mathbb{E}\left[M_{k, N, T}(X)\right]$.

$\triangleright$ We note that any increment $Y_{t}-Y_{s}$ of the delampertized $\mathrm{fBm}$, for $(s, t) \in \mathbb{R}^{2}$, is a Gaussian variable of mean 0 and of variance:

$$
\begin{aligned}
\mathbb{E}\left[\left(Y_{t}-Y_{s}\right)^{2}\right] & =e^{-2 \theta H t} \mathbb{E}\left[X_{\exp (\theta t)}^{2}\right]+e^{-2 \theta H s} \mathbb{E}\left[X_{\exp (\theta s)}^{2}\right]-2 e^{-\theta H(t+s)} \mathbb{E}\left[X_{\exp (\theta t)} X_{\exp (\theta s)}\right] \\
& =\sigma^{2}+\sigma^{2}-\sigma^{2} e^{-\theta H(t+s)}\left(e^{2 \theta H t}+e^{2 \theta H s}-\left|e^{\theta t}-e^{\theta s}\right|^{2 H}\right) \\
& =\sigma^{2}\left[2-e^{\theta H(t-s)}\left(1+e^{-2 \theta H(t-s)}-\left|1-e^{-\theta(t-s)}\right|^{2 H}\right)\right],
\end{aligned}
$$

thanks to equation (1). Increments are therefore stationary Gaussian variables and their variance, for the time step $1 / N$, is, for $H \in(0,1)$ :

$$
\mathbb{E}\left[\left(Y_{t}-Y_{t-1 / N}\right)^{2}\right]=\sigma^{2}\left[2-e^{\theta H / N}\left(1+e^{-2 \theta H / N}-\left|1-e^{-\theta / N}\right|^{2 H}\right)\right] .
$$

Then, using equation (4), we get:

$$
\mathbb{E}\left[\left(Y_{t}-Y_{t-1 / N}\right)^{k}\right]=\frac{2^{k / 2} \Gamma\left(\frac{k+1}{2}\right)}{\Gamma\left(\frac{1}{2}\right)} \sigma^{k}\left[2-e^{\theta H / N}\left(1+e^{-2 \theta H / N}-\left|1-e^{-\theta / N}\right|^{2 H}\right)\right]^{k / 2} .
$$

Again, by linearity, the same result holds for $\mathbb{E}\left[M_{k, N, T}(Y)\right]$.

$\triangleright$ When $\frac{\theta}{N} \rightarrow 0$, we have, as $H \in(0,1)$ :

$$
\begin{aligned}
\mathbb{E}\left[M_{k, N, T}(Y)\right] & =A(\sigma, k)\left[2-\left(1+\frac{\theta H}{N}+\mathcal{O}\left(\frac{\theta}{N}\right)^{2}\right)\left(2-\frac{2 \theta H}{N}+\mathcal{O}\left(\frac{\theta}{N}\right)^{2}-\left(\frac{\theta}{N}\right)^{2 H}\left(1+\mathcal{O}\left(\frac{\theta}{N}\right)\right)\right)\right]^{k / 2} \\
& =A(\sigma, k)\left[\left(\frac{\theta}{N}\right)^{2 H}+\mathcal{O}\left(\frac{\theta}{N}\right)^{\min (2 H+1,2)}\right]^{k / 2} \\
& =A(\sigma, k)\left(\frac{\theta}{N}\right)^{k H}\left[1+\mathcal{O}\left(\frac{\theta}{N}\right)^{\min (1,2-2 H)}\right]^{k / 2} \\
& =A(\sigma, k)\left(\frac{\theta}{N}\right)^{k H}+\mathcal{O}\left(\frac{\theta}{N}\right)^{k H+\min (1,2-2 H)} .
\end{aligned}
$$




\section{Proof of the propositions of Section 3.2}

Proof. The proof of the formulae concerning covariance and correlation in Proposition 1 and 2 is straightforward using Theorem 1 and the bilinearity of the covariance. The asymptotic results are a consequence of the asymptotic behaviour of the function $h$. For this purpose, we show that, for $x \rightarrow+\infty$ :

$$
\left(e^{x}-e^{-x}\right)^{2 H}=e^{2 H x}\left(1-2 H e^{-2 x}+o\left(e^{-2 x}\right)\right)
$$

Therefore, when $x \rightarrow+\infty$,

$$
\begin{aligned}
h(x) & =e^{H x}+e^{-H x}-\left(e^{x / 2}-e^{-x / 2}\right)^{2 H} \\
& =e^{-H x}+2 H e^{(H-1) x}+o\left(e^{(H-1) x}\right),
\end{aligned}
$$

or also $h(x)=\mathcal{O}\left(e^{\xi x}\right)$, where $\xi=\max (-H, H-1)$. As $\xi \in(-1,0), h(x)$ tends toward zero when $x$ tends toward infinity. This proves the asymptotic results in Proposition 1.

Concerning Proposition 2, we distinguish two cases:

$\triangleright$ If $H \leq 1 / 2$, then, for $x \rightarrow+\infty$, we have, from equation (5), $h(x)=e^{-H x}+\mathcal{O}\left(e^{(H-1) x}\right)$, so that

$$
\rho(s, d)=\frac{e^{-d H \theta s}\left(2-e^{H \theta s}-e^{-H \theta s}\right)+\mathcal{O}\left(e^{d(H-1) \theta s}\right)}{4-2 h(\theta s)}
$$

when $d \rightarrow+\infty$.

$\triangleright$ If $H>1 / 2$, then, for $x \rightarrow+\infty$, we have, from equation (5), $h(x)=2 H e^{(H-1) x}+o\left(e^{(H-1) x}\right)$, so that

$$
\rho(s, d)=\frac{2 H e^{d(H-1) \theta s}\left(2-e^{-(H-1) \theta s}-e^{(H-1) \theta s}\right)+o\left(e^{d(H-1) \theta s}\right)}{4-2 h(\theta s)}
$$

when $d \rightarrow+\infty$.

These two cases directly lead to the equivalence formulae provided in Proposition 2.

\section{References}

[1] Bardet, J.-M. And Surgailis, D. (2013), Nonparametric estimation of the local Hurst function of multifractional Gaussian processes, Stochastic processes and their applications, 123, 3: 1004-1045

[2] Bekiros, S. and Marcellino, M. (2013), The multiscale causal dynamics of foreign exchange markets, Journal of international money and finance, 33: 282-305

[3] Bouchaud, J.-P., Ciliberti, S., Lempérière, Y., Majewski, A., Seager, P. And Sin Ronia, K. (2017), Black was right: Price is within a factor 2 of value, arXiv preprint 1711.04717

[4] Brody, D.C., Syroka, J. And Zervos, M. (2002), Dynamical pricing of weather derivatives, Quantitative finance, 2, 3: 189-198 
[5] Caporale, G.M. and Gil-Alana, L.A. (2013), Long memory and fractional integration in high frequency data on the US dollar/British pound spot exchange rate, International review of financial analysis, 29: 1-9

[6] Cheridito, P., Kawaguchi, H. and Maejima, M. (2003), Fractional Ornstein-Uhlenbeck processes, Electronic journal of probability, 8, 3: 1-14

[7] Cheung, Y.W. (1993), Long memory in foreign-exchange rates, Journal of business \& economic statistics, 11, 1: 93-101

[8] Chevillard, L. (2017), Regularized fractional Ornstein-Uhlenbeck processes and their relevance to the modeling of fluid turbulence, Physical review E, 96, 3: 033111

[9] Chronopoulou, A. And Viens, F.G. (2012), Estimation and pricing under long-memory stochastic volatility, Annals of finance, 8, 2-3: 379-403

[10] Coeurjolly, J.-F. (2005), Identification of multifractional Brownian motion, Bernoulli, 11, 6: $987-1008$

[11] Cont, R. (2005), Long range dependence in financial markets, In Fractals in engineering (pp. 159-179), Springer, London

[12] Das, A. And Das, P. (2007), Chaotic analysis of the foreign exchange rates, Applied mathematics and computation, 185, 1: 388-396

[13] Diaz, J.F. And Chen, J.H. (2017), Testing for long-memory and chaos in the returns of currency exchange-traded notes (ETNs), Journal of applied finance and banking, 7, 4: 15-37

[14] Flandrin, P., Borgnat, P. and Amblard, P.-O. (2003), From stationarity to selfsimilarity, and back: Variations on the Lamperti transformation, Processes with long-range correlations, Springer, Berlin Heidelberg: 88-117

[15] Garcin, M. (2017), Estimation of time-dependent Hurst exponents with variational smoothing and application to forecasting foreign exchange rates, Physica A: statistical mechanics and its applications, 483: 462-479

[16] Garcin, M. (2018), Forecasting with a Hurst exponent lower than 1/2, working paper

[17] Garcin, M. And GuÉgan, D. (2016), Wavelet shrinkage of a noisy dynamical system with non-linear noise impact, Physica D: nonlinear phenomena, 325: 126-145

[18] He, K., Chen, Y. And Tso, G.K. (2018), Forecasting exchange rate using variational mode decomposition and entropy theory, Physica A: statistical mechanics and its applications, 510: $15-25$

[19] Hu, Y. and Nualart, D. (2010), Parameter estimation for fractional Ornstein-Uhlenbeck processes, Statistics \& probability letters, 80, 11-12: 1030-1038

[20] Kristoufek, L. (2018), Are the crude oil markets really becoming more efficient over time? Some new evidence, to appear in Energy economics

[21] Lamperti, J. (1962), Semi-stable stochastic processes, Transactions of the American mathematical society, 104, 1: $62-78$

[22] Lee, M., Genton, M.G. And Jun, M. (2016), Testing self-similarity through Lamperti transformations, Journal of agricultural, biological, and environmental statistics, 21, 3: 426447 
[23] Li, D., Kou, Z. And Sun, Q. (2015), The scale-dependent market trend: Empirical evidences using the lagged DFA method, Physica A: statistical mechanics and its applications, 433: 26-35

[24] LiU, G. AND Jing, B.Y. (2018), On estimation of Hurst parameter under noisy observations, Journal of business \& economic statistics, 36, 3: 483-492

[25] Mandelbrot, B. And van Ness, J. (1968), Fractional Brownian motions, fractional noises and applications, SIAM review, 10, 4: 422-437

[26] Mielniczuk, J. And WojdyŁeo, P. (2007), Estimation of Hurst exponent revisited, Computational statistics \& data analysis, 51, 9: 4510-4525

[27] Mitra, S.K. (2012), Is Hurst exponent value useful in forecasting financial time series?, Asian social science, 8, 8: 111-120

[28] Ohanissian, A., Russell, J.R. And Tsay, R.S. (2008), True or spurious long memory? A new test, Journal of business \& economic statistics, 26, 2: 161-175

[29] Peltier, R.F. And LÉvy VÉHel, J. (1994), A new method for estimating the parameter of fractional Brownian motion, Technical report 2396, INRIA

[30] Peng, C.K., Havlin, S., Stanley, H.E. and Goldberger, A.L. (1995), Quantification of scaling exponents and crossover phenomena in nonstationary heartbeat time series, Chaos: an interdisciplinary journal of nonlinear science, $5,1:$ : 82-87

[31] Pianese, A., Bianchi, S. and Palazzo, A.M. (2018), Fast and unbiased estimator of the time-dependent Hurst exponent, Chaos: an interdisciplinary journal of nonlinear science, 28, 3: 031102

[32] Ramos-Requena, J.P., Trinidad-Segovia, J.E. and Sánchez-Granero, M.A. (2017), Introducing Hurst exponent in pair trading, Physica A: statistical mechanics and its applications, 488: $39-45$

[33] Šapina, M., Garcin, M., Kramarić, K., Milas, K., Brdarić, D. and Pirić, M. (2017), The Hurst exponent of heart rate variability in neonatal stress, based on a mean-reverting fractional Lévy stable motion, working paper

[34] Šapina, M., Kośmider, M., Kramarić, K., Garcin, M., Pirić, M., Milas, K. and BrDARIĆ, D. (2018), Asymmetric detrended fluctuation analysis in neonatal stress, Physiological measurement, 39, 8: 085006

[35] Shittu, O.I. And YAya, O.S. (2009), Measuring forecast performance of ARMA and ARFIMA models: An application to US Dollar/UK pound foreign exchange rate, European journal of scientific research, 32, 2: 167-176

[36] Takaishi, T. (2018), Statistical properties and multifractality of Bitcoin, Physica A: statistical mechanics and its applications, 506: 507-519 